$10 \mid 2006$

Varia

\title{
Le sanctoral clunisien (xe-Xve siècles)
}

Thèse de doctorat de l'Université de Paris I-Panthéon-Sorbonne sous la direction de Michel Parisse, décembre 2005

\section{Catherine Bonnin-Magne}

\section{(2) OpenEdition}

\section{Journals}

Édition électronique

URL : https://journals.openedition.org/cem/316

DOI : $10.4000 /$ cem.316

ISSN : 1954-3093

Éditeur

Centre d'études médiévales Saint-Germain d'Auxerre

Édition imprimée

Date de publication : 15 août 2006

ISSN : 1623-5770

Référence électronique

Catherine Bonnin-Magne, «Le sanctoral clunisien (xe-xve siècles) », Bulletin du centre d'études médiévales d'Auxerre / BUCEMA [En ligne], 10 | 2006, mis en ligne le 12 septembre 2006, consulté le 22 septembre 2022. URL : http://journals.openedition.org/cem/316; DOI : https://doi.org/10.4000/cem. 316

Ce document a été généré automatiquement le 22 septembre 2022.

\section{c) (1)(2)}

Creative Commons - Attribution - Pas d'Utilisation Commerciale - Partage dans les Mêmes Conditions 4.0 International - CC BY-NC-SA 4.0

https://creativecommons.org/licenses/by-nc-sa/4.0/ 


\section{Le sanctoral clunisien (xe-Xve siècles)}

Thèse de doctorat de l'Université de Paris I-Panthéon-Sorbonne sous la direction de Michel Parisse, décembre 2005

\section{Catherine Bonnin-Magne}

1 Par sanctoral, nous entendons l'ensemble des saints fêtés à Cluny dont le nom est inscrit dans les calendriers ou dans le propre des saints des livres liturgiques. La liste de ces saints peut être établie grâce aux calendriers, généralement annexés aux livres liturgiques, ainsi qu'aux textes du propre des saints de ces mêmes livres. L'objectif premier du travail a été d'établir le sanctoral propre à l'abbaye de Cluny et d'en reconstituer la mise en place et l'évolution entre les $\mathrm{x}^{\mathrm{e}}$ et $\mathrm{Xv}^{\mathrm{e}}$ siècles.

2 Ce choix d'enquête a été important à plus d'un titre : tout d'abord, il m'a conduit sur la piste de la source principale de cette recherche puisque c'est en cherchant des calendriers que j'ai été confrontée, pour la première fois, au matériau qui guide ce travail: les livres liturgiques. Ensuite, ce choix a fait naître un certain nombre d'interrogations quant à la constitution et l'évolution du sanctoral de Cluny et quant à l'apport de l'analyse du sanctoral pour les études clunisiennes. Quels étaient donc les saints fêtés dans le calendrier ? Y avait-il là une particularité clunisienne ? Ces interrogations ne trouvaient pas de réponse complète chez les historiens qui se sont intéressés à divers titres au sanctoral clunisien ${ }^{1}$.

3 À l'origine, les questions posées s'articulaient autour de la problématique de la particularité ou de "l'identité" du sanctoral. L'étude des saints fêtés dans un lieu me semblait poursuivre plusieurs types de questionnements à l'épreuve des sources: considérer conjointement les différents calendriers et en extraire les éléments originaux de l'identité clunisienne. D'une part, il me fallait poser la question du lien entre l'évolution du calendrier et l'histoire de l'abbaye de Cluny. D'autre part, ce travail voulait envisager le rôle du sanctoral dans l'ecclesia cluniacensis, en posant le problème de savoir si l'abbaye de Cluny avait imposé un sanctoral propre à ses dépendances et si cette imposition avait varié à l'intérieur de l'ecclesia puis de l'ordo cluniacensis. Enfin, l'enquête ne pouvait être complète, sur le plan liturgique, sans la reconstitution du 
culte de chacun des saints propres à Cluny. Pour répondre à ces questions, il fallait choisir un terrain d'enquête adapté.

Un terrain chronologique tout d'abord : c'est la période allant des origines jusqu'au $\mathrm{xv}^{\mathrm{e}}$ siècle qui s'est immédiatement imposée comme une période clé; en effet les sources sont rares et tardives si l'on ne s'intéresse qu'à l'abbaye de Cluny, car nous ne possédons aucune source directe du calendrier antérieur au XIII ${ }^{e}$ siècle. Ensuite, l'intérêt d'englober l'après-Cluny', c'est-à-dire l'époque où la congrégation clunisienne ou ecclesia cluniacensis s'est transformée en un ordre structuré, permettait de poser la question de la transmission du sanctoral et du renforcement de la tendance à l'uniformisation sur le temps long ${ }^{2}$. Enfin, l'apparition des premiers livres imprimés, à la fin $\mathrm{du} \mathrm{Xv}^{\mathrm{e}}$ siècle, constituait une borne naturelle pour cette période.

5 Mais pour servir le projet et porter attention à la transmission du calendrier vers les dépendances, il fallait aussi délimiter un terrain géographique, afin de donner corps à cette histoire. Mon choix s'est porté sur quelques dépendances phares pour lesquelles plusieurs sources du sanctoral sont conservées. Le choix de ces dépendances se justifiait par l'importance, la diversité des sources rencontrées et le caractère spécifique de chacune d'elles. Que ce soit Moissac, Saint-Martial de Limoges ou SaintBenoît de Polirone, elles occupent en majorité une place importante dans la hiérarchie clunisienne et contrôlent leur propre réseau de dépendances. De plus, ces prieurés présentaient l'atout d'offrir l'exemple d'abbayes bien documentées, ce qui me permettait d'avancer sur un terrain quelque peu balisé. Or ce choix s'est avéré, en pratique, intéressant car le foisonnement documentaire qui caractérise Saint-Martial de Limoges ou Saint-Pierre de Moissac m'a permis de réfléchir aux types de liens qui s'établissaient entre les dépendances et l'abbaye-mère.

6 Car d'emblée l'approche fut élargie. En effet, je ne pouvais compter sur une histoire du calendrier et du sanctoral de Cluny sans l'analyse du calendrier des dépendances, il était parfois difficile de distinguer ce qui vient de Cluny de ce qui est clunisien. Or cette difficulté s'est rapidement instituée comme un défi : n'était-il pas possible, dès lors, de comprendre, grâce au degré d'adoption du calendrier, une certaine différenciation dans les modalités de son adoption selon le rapport entretenu à Cluny : la plus ou moins grande adhésion au modèle de Cluny correspondait-elle à la quête d'une plus grande autonomie? Le sujet engageait alors nécessairement une démarche multiple, déclinée sur plusieurs tableaux: il fallait reconstituer le calendrier de cluny et celui des dépendances à diverses périodes; comparer les différences d'adoption et de transmission du sanctoral selon les dépendances en rapport avec le degré d'adhésion et de fidélité à l'abbaye bourguignonne; réfléchir à la façon dont ont été fêtés les saints abbés, en étudiant les différents formulaires et en étant attentive aux compositions originales.

7 Cette recherche aurait pu se contenter, dans une approche historique, d'exploiter les données fournies par les calendriers, sources riches et précieuses bien que relativement rébarbatives et arides. Il s'est agi de dépouiller un ensemble conséquent de calendriers et propres des saints issus des bréviaires et missels ainsi que des coutumiers et statuts qui parfois témoignent de l'introduction d'un nouveau culte. Ce travail fastidieux de prime abord s'est avéré tout à fait passionnant, puisqu'il permettait de comprendre l'enrichissement progressif du calendrier de Cluny. Le calendrier, en effet, loin d'être un document figé et à part, reflète l'histoire d'un lieu. 
8 Dans un premier temps, il fallait reconstituer une chronologie afin d'apercevoir sur le long terme les évolutions du sanctoral en rapport avec la transformation de l'ecclesia cluniacensis en un ordre bien structuré. Il fallait s'armer de patience pour analyser les données collectées dans les différents calendriers. Pourtant, il me fallait utiliser ces données non pour elles-mêmes mais pour répondre à mes questions. Or le nombre des inscriptions relevées dans le calendrier (plus de 400 noms au final ont été saisis), montrait, au fil du travail, que l'analyse chronologique présentait des insuffisances. Comment comprendre le sens et la nature des différents saints entrés dans le calendrier à partir des données collectées ? Travailler sur des calendriers m'a conduit à la conviction qu'une approche uniquement chronologique et quantitative (stratigraphique) ne suffisait pas à rendre compte des logiques à l'œuvre dans la confection d'un calendrier commun.

9 La seconde méthode qui consiste à récolter les sources disponibles sur des prieurés à partir de leur liste de saints, s'est avérée particulièrement fructueuse. Listes nominatives de pitances, listes de lectures, ou encore inventaires de reliques et récits de translations... Autant de dossiers qui aident à comprendre le phénomène d'enrichissement $d u$ calendrier et de transmission du sanctoral. Certes, il a fallu restreindre, pour cette partie du travail, le terrain d'analyse. Mon choix s'est porté sur l'étude des litanies et les lectures du sanctoral. Pourquoi ? Ces sujets offraient, à mes yeux, une illustration concrète de la manière dont s'effectuait la "clunification" d'un prieuré. La réflexion m'amenait alors à découvrir l'intérêt d'une approche plus historique (sociale) de mon objet (le sanctoral et le calendrier). Une histoire du sanctoral clunisien qui comporte à la fois une histoire de la mise en place d'une liste de saints identitaire d'une part, et une histoire de la transmission de ce sanctoral, d'autre part; enfin une histoire du culte des quatre saints abbés de Cluny qui constituent le sommet de ce calendrier. Il s'agissait de faire le lien entre ces processus et travailler sur le calendrier des saints comme moyen d'acculturation et d'uniformisation.

10 Cette méthode a été appliquée à la liturgie des saints de Cluny: ici encore il a fallu choisir, et le choix s'est porté sur les saints abbés de Cluny, excluant ainsi, peut-être un peu rapidement, les autres saints typiques du calendrier. Porter l'accent sur les quatre abbés conduit à évaluer leur statut et leur position dans l'ecclesia cluniacensis puis dans l'ordo cluniacensis à l'aune de leur culte liturgique et c'est l'objet de la deuxième partie de la thèse : tenter de suivre les cheminements qui mènent de l'insertion d'un nouveau nom dans le calendrier jusqu'à la mise en place et la diffusion d'un culte dans ses multiples aspects.

Évolution du sanctoral

11 Une première étape a été de montrer comment s'est effectué l'enrichissement du sanctoral en mettant en évidence les différentes strates successives qui l'ont constitué. Pour connaître le sanctoral d'un lieu, le plus sûr moyen est de s'en rapporter au calendrier qui est le témoin le plus visible du culte des saints et aussi le plus facile à appréhender, puisqu'une suite de calendriers reflète le développement du sanctoral et permet de reconstituer les principales étapes de sa mise en place.

12 En relevant les noms inscrits dans les calendriers ou en étudiant les propres des saints on a pu dresser une liste des saints, souvent longue, célébrés tout au long de l'année. Cette liste n'est pas ici analysée tout entière, mais nous y avons recherché les saints propres en mettant de côté ceux qui appartiennent au commun dénominateur, les 
saints universaux présents dans bon nombre de calendriers, afin de se concentrer sur l'introduction de saints régionaux, plus significatifs pour notre propos.

Les témoins les plus anciens montrent un sanctoral fortement carolingien avec une empreinte bénédictine. Un sanctoral qui est encore peu particularisé, même si à travers lui, l'histoire des origines de Cluny et les dévotions des premiers abbés se dessinent (les saints apôtres, Pierre et Paul, patron de l'abbaye, la Vierge et les saints Lautein, Martin, Géraud). C'est avec Odilon que le sanctoral clunisien semble prendre un premier caractère propre avec les saints abbés de Cluny, Odon et surtout Maïeul, figure majeure de l'ecclesia cluniacensis naissante, et les saints dont l'abbaye possède des reliques (les papes Marcel et Grégoire, Consorce). Avec Hugues, l'augmentation du sanctoral est considérable: la lignée abbatiale s'affirme avec la reconnaissance officielle de la sainteté d'Odilon par le légat pontifical Pierre Damien, le calendrier s'enrichit aussi d'une multitude de saints qui reflètent les relations entretenues avec d'autres abbayes ou qui rappellent un saint vénéré dans une abbaye entrée dans le réseau des dépendances clunisiennes. Le sanctoral de Cluny qui s'est profondément modifié pendant les abbatiats d'odilon et d'Hugues (passant d'une quarantaine à plus de 260 fêtes inscrites) se stabilise à l'époque de Pierre le Vénérable (saint Hugues est canonisé en 1121); au cours des XIII et $\mathrm{XV}^{\mathrm{e}}$ siècles, il ne s'enrichit guère, hormis quelques nouvelles translations de reliques et l'instauration par des statuts de la fête de deux prieurs de Cluny contemporains de l'abbé Hugues.

Les contextes historique et liturgique modèlent bien évidemment ces calendriers qui sont éminemment réceptifs aux apports extérieurs : l'insertion d'un nouveau saint, la translation de reliques ou l'aménagement de l'église sont à l'origine de l'inscription d'une nouvelle fête. On le voit bien lorsque, à la suite d'un décret instituant un nouveau culte ou à la suite d'une arrivée de reliques, on assiste à la généralisation de l'inscription d'une fête dans les différents calendriers clunisiens. Retracer les flux d'inscriptions nouvelles dans le calendrier au regard des changements structurels de la congrégation clunisienne permet de montrer que c'est moins l'origine d'un saint qui détermine les modes d'insertion d'un nouveau culte que les influences extérieures ou les instantanés d'histoire (confraternité, échanges de reliques, nouvelle dépendance).

Sanctoral et dépendances

Il est parfois difficile de faire la part entre l'apport propre à l'abbaye bourguignonne et l'apport des dépendances, d'autant plus que l'évolution du calendrier de Cluny est en partie reconstituée grâce à celui de ses dépendances. Cependant il apparaît nettement qu'il existe un modèle commun, élaboré à Cluny. Que ce soit à Saint-Martial de Limoges, à Saint-Pierre de Moissac, à Saint-Arnoul, à L'Échelle-Saint-Taurin, à La Charité-surLoire, à Saint-Pancrace de Lewes... Les calendriers et propres des saints des bréviaires et missels comportent une même liste de saints. Cet ensemble de fêtes, qu'on appelle pour plus de facilité la liste clunisienne du sanctoral, constitue le fonds commun à tous les calendriers de l'ecclesia cluniacensis.

Il fallait montrer comment ces entrées dans l'ecclesia cluniacensis s'inscrivaient historiquement et liturgiquement dans le calendrier et les usages pratiqués dans la dépendance. La liste clunisienne du sanctoral s'enrichit des fêtes propres à un lieu, et dans un curieux brassage, les solennités locales se retrouvent aux côtés des saints clunisiens importés, symbole des nouveaux liens. L'entrée dans l'ecclesia cluniacensis supposait bien plus que l'adoption d'un simple calendrier conforme à celui de la maison-mère, elle impliquait également l'adoption d'une liturgie conforme aux 
pratiques et à l'ordo, de l'abbaye-mère. La transmission d'une liste clunisienne de saints, visible à travers l'étude des litanies et des lectures communes, démontre une mise aux normes liturgiques consécutive à l'entrée dans l'orbite clunisienne. Les saints invoqués dans les litanies sont les mêmes que ceux qui sont inscrits dans les calendriers de Cluny. Les différentes listes de lectures, que celles-ci aient lieu à l'église, au réfectoire ou au chapitre, présentent une organisation et des choix de textes semblables; ces lectures qui réunissaient l'ensemble du convent sont la base d'une culture clunisienne commune à l'ensemble des membres de la congrégation clunisienne ${ }^{3}$.

Saints abbés

17 La seconde partie est tout entière consacrée à l'étude, dans le détail, du culte liturgique des quatre saints abbés de Cluny, qui sont la marque du sanctoral clunisien. La reconstitution, effectuée à partir des plus anciens témoins du sanctoral du culte liturgique des abbés de Cluny jusqu'à ceux plus récents $d u \mathrm{xv}^{\mathrm{e}}$ siècle, doit être suffisamment minutieuse pour qu'on puisse avoir une idée exacte du contenu des différents formulaires et de son évolution.

Dans un premier temps pour chacun des quatre abbés, on s'attache à reprendre les éléments du dossier du saint, en insistant sur les différentes fêtes et leur explication (le plus souvent une translation de reliques) et les efforts faits par les abbés eux-mêmes pour promouvoir le culte de leur prédécesseur.

Le modèle suivi pour fêter les saints abbés de Cluny reprend les formulaires des différentes fêtes de saint Benoît transmis dans les sacramentaires; cet emprunt dénote la volonté d'inscrire les saints abbés de Cluny dans la lignée bénédictine. Les abbés Maïeul et Odilon sont les seuls pour lesquels nous soient parvenus des offices complets et originaux qui ont été vraisemblablement composés à Cluny; l'étude des offices des saints Maïeul et Odilon montre une étroite dépendance à l'égard du dossier hagiographique de ces saints. Si quelques offices ont été composés à Cluny ou à Souvigny, l'effort principal des abbés a porté sur la reconnaissance de la sainteté de leur devancier et sur la composition et la diffusion d'un ensemble de textes hagiographiques regroupant les Vies des saints abbés ${ }^{4}$.

La dernière partie de la thèse s'attache ainsi à montrer le processus qui aboutit à l'édification d'un modèle commun de sainteté clunisienne. L'utilisation d'un formulaire commun et uniforme s'accentue en parallèle à la transformation de la congrégation clunisienne en une structure contraignante (ordo cluniacensis). Le processus d'uniformisation liturgique contribue ainsi à réduire amplement le principe de la production originale qui semblait prévaloir aux débuts de l'histoire clunisienne (Odon, Odilon, hagiographes) et il participe à l'unification progressive de l'ordre.

Au terme de cette enquête plusieurs éléments de réponse peuvent être avancés :

Cluny a largement diffusé un sanctoral, dont on pourrait reconstituer le prototype ; cependant cette transmission vers les dépendances n'est pas une imposition brutale, il laisse la place à une adaptation locale. Le sanctoral résulte aussi d'un échange, entre Cluny et ses dépendances. Chaque monastère conservait en effet sa propre tradition locale et pouvait enrichir le calendrier des saints vénérés localement et le propre des saints des bréviaires et lectionnaires était ouvert aux apports liturgiques et culturels propres à un lieu. Ainsi c'est le mélange et le syncrétisme qui s'imposèrent. 
Le calendrier est une marque d'appartenance. Il est avec les coutumiers, qui définissent les usages et pratiques quotidiennes, les offices monastiques et le cycle liturgique, un élément constitutif de l'identité clunisienne.

Le sanctoral, tout comme l'hagiographie clunisienne et la réécriture des origines, participe à l'affirmation de l'ecclesia cluniacensis comme structure indépendante et place Cluny dans la légitimité apostolique et bénédictine. Le calendrier est un facteur d'intégration de premier ordre, il donne force à l'idée d'un corps unique, composé d'une tête, Cluny, et d'une multitude de membres, les prieurés. Les saints abbés de Cluny sont les symboles de l'institution ecclésiastique qu'ils représentent. Un calendrier et un sanctoral communs participent, tout comme le martyrologe, le nécrologe et les fraternisations au processus d'unification, ils soudent les membres et la tête de la communauté monastique.

S'il fallait résumer d'un mot le parti pris qui a guidé ce travail, c'est bien de chercher des indicateurs pertinents capables de mettre au jour les rapports entre calendrier et histoire, les normes et les pratiques. Dans ce projet, l'enjeu consiste bien évidemment à travailler à l'intersection des disciplines, empruntant notions et méthodes tant à l'histoire qu'à la liturgie, pour étudier la place du sanctoral dans l'histoire clunisienne. J'espère avoir montré dans cette thèse combien cet échange était nécessaire et, comment à partir du prisme de départ très pointu, le sanctoral, on pouvait atteindre l'histoire clunisienne, et, sans l'a priori d'y trouver des choses nouvelles ou originales, apporter des éléments complémentaires qui permettaient de mieux cerner la chronologie clunisienne et éclairer ce passage d'une congrégation lâche à un ordre.

\section{NOTES DE FIN}

1. Plusieurs études abordent la question du calendrier clunisien : Guy de valous, Le monachisme clunisien des origines au XVe siècle, Paris, 1935, 2 volumes, p. 397-410 ; R. ETAIX, «Le lectionnaire de l'office à Cluny ", in Recherches Augustiniennes, 11 (1976), $\mathrm{n}^{\circ}$ 190, p. 91-159 ; Dom K. HALLINGER, « Das Sanktorale Klunys nach der Mitte des 11. Jahrhundert ", Corpus consuetudinum monasticarum, VII/1-3, 1984, p. 19-35 ; R. HAUSMANN, « Das Martyrologium von Marcigny-sur-Loire », in Hochschulsammlung Philosophie Geschichte, Band 7, Fribourg, 1984, p. 32-113 ; P. HENRIET, « Sanctoral clunisien et sanctoral hispanique au XIIe siècle, ou de l'ignorance réciproque au syncrétisme. À propos d'un lectionnaire de l'office originaire de Sahagún (fin XIIe siècle), in É. RENARD, M. TRIGALET, X. BERMAND ET P. BERTRAND (éd.), Scribere sanctorum gesta. Recueil d'études d'hagiographie médiévale offert à Guy Philippart, Hagiologia, vol. 3, Turnhout, 2005, p. 209-261.

2. G. MELVILLE, « Cluny après "Cluny". Le treizième siècle : un champ de recherches ", in Francia, 17/1 (1990), p. 91-124.

3. Sur les différentes lectures monastiques, voir D. NEBBIAI-DALLA GUARDA, « Les listes médiévales de lectures monastiques. Contribution à la connaissance des anciennes bibliothèques bénédictines ", in Revue bénédictine, 96 (1986), p. 271-323, en particulier 
p. 298 ; A. DEROLEZ, Les catalogues de bibliothèques, Turnhout, 1979 (Typologie des sources du Moyen Age, 31) ; D. B. GRÉMOND, « Les lectiones ad prandium à l'abbaye de Fécamp au XIIIe siècle », in Cahiers Léopold Delisle, 1971, p. 3-41 et J. LECLERCQ, « Un coutumier de Saint-Martial », in Revue Mabillon, 44 (1954), p. 40-43.

4. Le dossier hagiographique des saints abbés est bien connu, on peut se reporter à

D. IOGNA-PRAT, Études clunisiennes, Paris, 2002.

INDEX

Mots-clés : sanctoral

Index géographique : France/Cluny 